\title{
Development of a Wafer-Bonded, Silicon-Nitride Membrane Thermal Shear-Stress Sensor with Platinum Sensing Element
}

\author{
Anthony Cain ${ }^{\S}$, Venkataraman Chandrasekaran, Toshikazu Nishida and Mark Sheplak \\ Interdisciplinary Microsystems Group \\ University of Florida \\ Gainesville, Florida 32611-6250
}

\begin{abstract}
ABSTRAC'T
This paper presents the development of a thermal shearstress sensor for quantitative turbulence measurements. The shear-stress sensor was fabricated by an aggressive wafer bonding process that produces a $1500 A$-thick silicon nitride membrane over a vacuum cavity which supports a platinum sensing element. Static sensitivity data at multiple overheat ratios (11 $\mathrm{mV} / \mathrm{Pa}$ at an overheat of 1.0 ), pressure sensitivity spectra $(<1 \mu V / P a)$, noise floor spectra $(100 n V / \sqrt{/ H z})$ and direct, in-situ dynamic calibration data are presented. These calibrations were performed using a four-point probe configuration to exclude the effects of external bias circuitry and to isolate the sensor performance.
\end{abstract}

\section{INTRODUCTION}

Wall shear stress measurement within a turbulent boundary layer provides insight into complex flow phenomena, including skin friction, flow separation and reattachment. The mean component indicates the average state of the flow over a surface, and the dynamic component is a signature of the processes responsible for the unsteady momentum transfer to the wall [1]. The fluid structures of interest are dimensionally on the order of 100 micrometers, requiring a sensor clement of the same size to minimize spatial averaging. Also, a shear stress sensor with a usable bandwidth of over $10 \mathrm{kHz}$ is needed to accurately capture the complete spectrum of turbulent fluctuations [2].

Conventional shear stress measurement techniques can be broadly classified into two types: direct (e.g., floating element devices) and indirect (e.g., thermal sensors). Micromachined varieties of both of these types of sensors have been developed for turbulence measurements [3-6]. Thermal sensors [3,4] are generally simpler to fabricate and are more robust than floating element sensors $[5,6]$. However, these require an empirical correlation, valid for very specific conditions, to relate the Joulean heating rate to the induced shear stress [7]. Since the device operates on thermal transfer principles, the ratio of the convection of heat into the flow (used for shear stress transduction) and the various storage and dissipation mechanisms ultimately limit the dynamic response and the operational bandwidth. In particular, the unsteady heat conduction into the substrate results in a roll-off in the gain factor of the frequency response function (FRF), as well as a phase lag at low frequencies [8]. Therefore, these sensors require extensive in-situ calibration before they can be utilized for quantitative measurements in a flow field. Liu et al. [3] presented a novel polycrystalline silicon-based sensor on a membrane over a vacuum cavity to reduce substrate conduction effects. This device showed improved sensitivity over traditional devices, but the sensor operation was limited to moderate thermal overheats $(0.65)$, and an experimental verification of the FRF using a known shear-stress input was not reported.

In this work, a shear stress sensor is presented that employs a thin-film platinum sensing element on top of a silicon-nitride

$\$$ Anthony Cain is presently affiliated with the Air Force Research Laboratory, AFRL/MLPO, WPAFB, Dayton, OH 45433-7707. membrane. This membrane is stretched over a crrcular vacuum cavity with the goal of providing an extended thermal operating range, a lower noise floor, and reduced pressure sensitivity. Advantages of platinum-based sensors over polycrystalline silicon-based sensors include higher thermal coefficient of resistance (TCR), higher thermal operating range, reduced $1 / f$ noise [9], and no piezoresistive-induced pressure sensitivity.

The details of the fabrication process for the shear-stress sensor are provided and experimental results presented. Specifically, rigorous static and dynamic characterizations of the sensor are reported for a constant current (CC) mode of excitation. In addition, the experimental verification of potential error sources is provided, including dynamic pressure sensitivity and noise floor spectra.

\section{DESIGN CONSIDERATIONS}

Typical metals used for conventional hot-film and hot-wire sensors include tungsten, platinum, and platinum-iridium [10] It is desirable to use a material with a high temperature coefficient of resistance (TCR), high resistivity, and low thermal conductivity. The first two parameters will determine the sensitivity to velocity or shear stress and the latter parameter will affect the amount of heat that is conducted into the electrical contacts. Of the three metals, bulk platinum offers the second highest TCR, the highest resistivity, the highest operating temperature and the second lowest thermal conductivity. It is also favorable because it does not form an oxide. Due to these factors, platinum is widely considered to be the optimal metal for use as a hot-film sensor [10].

During operation, the sensing element is resistively heated to a temperature greater than the gas temperature defined by the non-dimensional thermal overheat ratio,

$$
a_{T}=\frac{T_{s}-T_{g}}{T_{g}}
$$

where $T_{s}$ and $T_{g}$ are the absolute sensor and gas temperatures, respectively. The convection of heat from the sensor is related to the wall shear stress by the Reynolds analogy [11] and is measured by monitoring changes in the temperature-dependent resistance of the sensing element:

$$
R_{s}=R_{r}\left[1+\alpha\left(T_{s}-T_{r}\right)\right],
$$

where $R_{s}$ is the sensor resistance, $T_{r}$ is a reference temperature corresponding to the sensor reference resistance, $R_{r}$, and $\alpha$ is the TCR. The sensor can be operated in one of three biasing modes: constant current (CC), constant voltage (CV), or constant temperature (CT). Constant current and constant voltage modes are open-loop systems that use constant current and voltage sources, rcspectively, to provide heating power to the sensor. These modes do not regulate sensor temperature, and thus the sensor resistance varies with changes in the fluid temperature and velocity. Consequently, when thermal overheat is specified for $\mathrm{CC}$ and $\mathrm{CV}$, it is the zero mean flow overheat relative to the initial fluid temperature. In comparison, the CT mode is a 
closed-loop system, providing heating power to the sensor to maintain a constant sensor temperature and thus resistance [10].

To accurately capture the complete spectrum of shear stress fluctuations in a turbulent flow, a sensor should possess a flat, zero-gain, minimum-phase frequency response. This dictates that the sensor have equal static and dynamic sensitivities over the frequency range of interest. In general, the inherent compliance, inertance and dissipation of the system limit the dynamic response. The limiting factors are thermal inertia of the substrate and the sensing element, which restrict the usable bandwidth of the sensor [12] and the unsteady heat conduction into the substrate. This further complicates the dynamic response. In order to minimize uncertainties in correlation and spectral analysis of the measured shear stress, an in-situ dynamic calibration technique is required to identify the frequency response function (FRF).

\section{SENSOR FABRICATION}

The device structure consists of a $1500 A$-thick $\times 4 \mu \mathrm{m}$ wide $\times 200 \mu \mathrm{m}$-long platinum sensing element on top of a 1500 $\not A$-thick silicon nitride membrane which seals a $200 \mu m$-diameter and $10 \mu m$-deep vacuum cavity. Figure 1 is a plan-view SEM of the active area of the shear-stress sensor. Two gold leads at each end of the sensing element permit 4-point probe characterization exclusive of the effects of the biasing circuitry. Titanium serves as an adhesion layer for the leads and sensing element. The shear-stress sensors were fabricated using a silicon-rich siliconnitride/silicon fusion-bond and thin-back process [13] outlined in Figure 2. The process begins with two p-type $\langle 100\rangle$ singlepolished silicon wafers. Cavities are etched via DRIE in the handle wafer followed by growth of $7000 A$ of thermal oxide as a bonding layer. The membrane material, $1500 A$ of silicon-rich silicon nitride, is deposited on the device wafer and polished via CMP in preparation for bonding (A). The wafers are bonded in $100 \% \mathrm{O}_{2}$ ambient and annealed at $1100{ }^{\circ} \mathrm{C}$ for one hour. A vacuum is formed within the cavities during the anneal as $\mathrm{O}_{2}$ is consumed by the growth of $\mathrm{SiO}_{2}$ [14]. The handle wafer is protected and the device wafer is thinned back to the silicon nitride using $20 \% \mathrm{KOH}$ at $60^{\circ} \mathrm{C}(\mathrm{B})$. The sensing element and leads are patterned via image reversal lithography and titanium, platinum, and gold are realized via E-beam deposition. The sensing element and leads are realized via lift-off and the gold is etched from the sensing element using a KI wet etch (C). This compact, three-mask process yielded strict diaphragm geometry control $( \pm 3 \%)$ for the vacuum cavity. Several die configurations were designed including 9-sensor arrays for wind tunnel testing and single sensor dics with and without vacuum cavitics for sensor characterization. Packaging for characterization experiments was accomplished by flush mounting the sensor dies within a Lucite plug with front to back electrical contacts. The sensor leads are ball-bonded to the package contacts using $0.001 \mathrm{in}$. gold wire and are oriented downstream of the sensor to minimize flow disruption during testing.

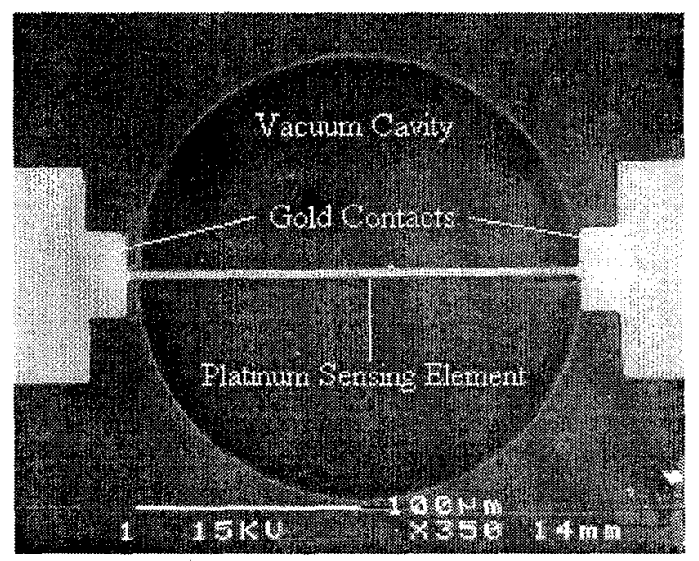

Figure 1. Plan-view SEM of the active area of the shear stress sensor.

(A)
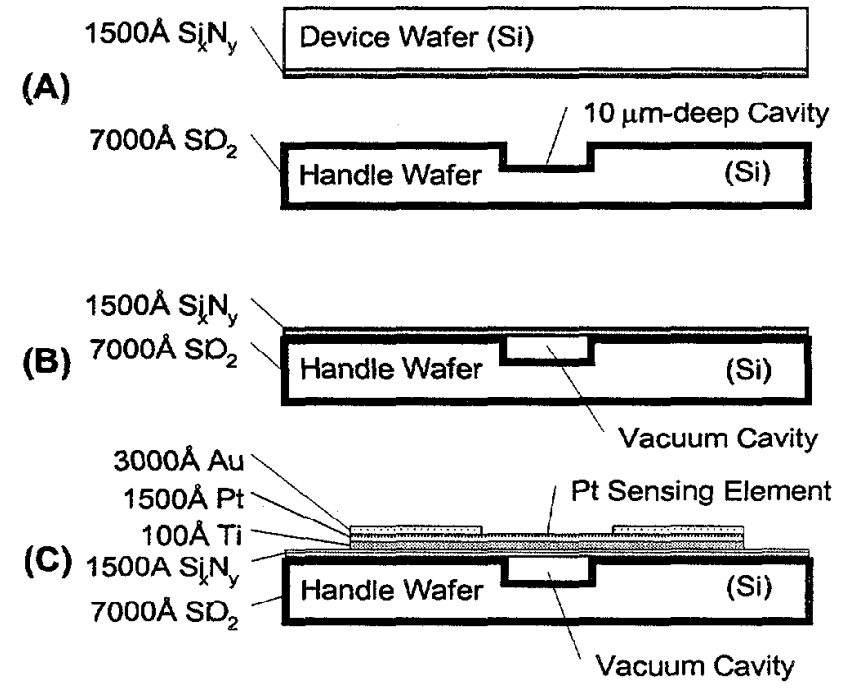

Figure 2. Cross-sectional schematic of the sensor fabrication sequence.

\section{RESULTS AND DISCUSSION}

All sensor measurements were performed using a 4-point probe configuration to exclude the effects of the external circuitry and the parasitic lead impedance. This entails maintaining a constant current through the sensing element and monitoring the varying voltage across the sensor using a Keithley 2400 source meter.

The temperature coefficient of resistance of the thin-film platinum resistor was experimentally determined using a hightemperature alumina substrate jig that permitted a 4-point resistance measurement of the sensor within a furnace. A TCR of $0.0029{ }^{\circ} \mathrm{C}^{-1}$ posscssing a maximum non-lincarity of $2.7 \%$ over a temperature range of $20{ }^{\circ} \mathrm{C}$ to $400{ }^{\circ} \mathrm{C}$ was observed (Figure 3 ). The effectiveness of the vacuum cavity in thermally isolating the sensing element from the substrate was investigated by comparing the sensor overheat as a function of input power for sensors with and without a cavity (Figure 4). The sensors with cavities exhibited much greater overheat than those without cavitics. The scnsors without cavities could only achieve a maximum overheat of 0.01 before exceeding a destructive current density limit of $1 \times 10^{7} \mathrm{~A} / \mathrm{cm}^{2}$. 


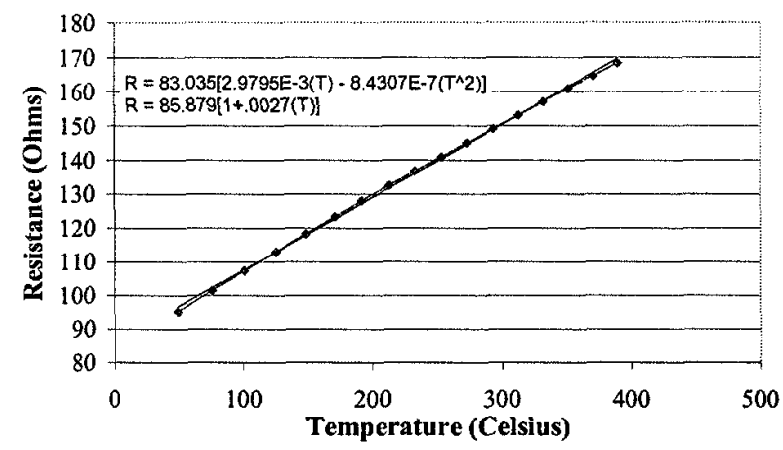

Figure 3. Resistance vs. temperature relationship for the thinfilm platinum sensing element.

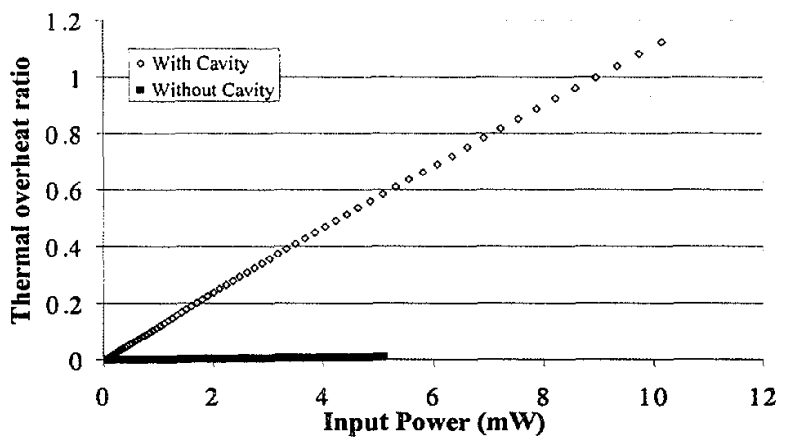

Figure 4. Thermal overheat ratio vs. input power for sensors with and without cavities.

Static wall shear stress sensitivity experiments were performed in $\mathrm{CC}$ mode of excitation using a laminar flow cell for thermal overheats of $0.2-1.0$ and wall shear stresses from 0 $\mathrm{Pa}-1.7 \mathrm{~Pa}$ (Figure 5). The static sensitivity increased with higher thermal overheat with a maximum sensitivity of 11 $\mathrm{mV} / \mathrm{Pa}$ at an overheat of 1.0. A lumped, two-dimensional power balance relationship for hot film sensors [15] is commonly used as the fitting equation for these calibrations. This relationship suggests a $1 / 3^{\text {rd }}$-power dependence of the sensor heating power on shear stress based on several major assumptions, most notably, that the thermal boundary layer lies within the linear region of the velocity boundary layer and the sensing element lies on a semi-infinite substrate. This device did not exhibit the $1 / 3^{\text {rd }}$-power dependence which is physically plausible since the sensor is located on a diaphragm over a vacuum cavity and not on a semi-infinite substrate.

Dynamic wall shear stress sensitivities were obtained at multiple mean shear stress levels and overheats, using a novel, in-situ dynamic calibration technique. This technique provides known sinusoidal shear-stress perturbations generated via acoustic plane-wave excitation $[16,17]$. These calibrations were performed in a constant-current mode of excitation for thermal overheats of $0.6-1.0$ and for mean shear stress levels of $0.03 \mathrm{~Pa}$ to $0.06 \mathrm{~Pa}$. A constant amplitude acoustic excitation of $105 \mathrm{~dB}$ SPL (ref. $20 \mu P a$ ) was used for the calibration. The dynamic shear stress FRF is a function of the static sensitivity as shown in Figure 6 but is independent of the overheat ratio as seen by the overlapping curves in Figure 7. An increase in dynamic sensitivity with higher thermal overheats was observed and in general, the sensor exhibited $\approx 40 \mathrm{~dB} /$ decade roll-off with a corner frequency of $\approx 600 \mathrm{~Hz}$, indicative of a highly damped $2^{\text {nd }}$ order system. Previous work [18] uses a 1/2-order system to model the response of a sensor on a semi-infinite medium. The difference in response is explained by the presence of a sealed vacuum cavity that drastically reduces the unsteady heat conduction into the substrate and limits the dissipation due to conduction losses into the thin membrane.

The dynamic range of the sensor is ultimately limited by its cross sensitivities to non-shear-stress inputs and the device noise floor. Experiments were performed to quantitatively measure both the pressure sensitivity and the noise floor. The dynamic pressure sensitivity spectrum was determined within a planewave tube by orienting the sensor normal to the propagation of the acoustic waves under zero mean flow conditions. Frequency sweeps at multiple sound pressure levels ranging from $120 \mathrm{~dB}$ to $155 d B$ (ref. $20 \mu P a$ ) at a constant overheat of 1.0 were used for the measurements. The results indicate negligible pressure sensitivity $(<1 \mu \mathrm{V} / \mathrm{Pa}$, Figure 8) up to $10 \mathrm{kHz}$. The noise floor spectra at zero mean flow and multiple overheats were also measured $(<100 \mathrm{nV} / \sqrt{\mathrm{Hz}})$ and is shown in Figure 9. Electromagnetic interference appears to be the major contributor at low frequencies with all harmonics decaying within $1 \mathrm{kHz}$. Any observed response of the sensor to pressure fluctuations may also be due to buoyant convection from the sensor.

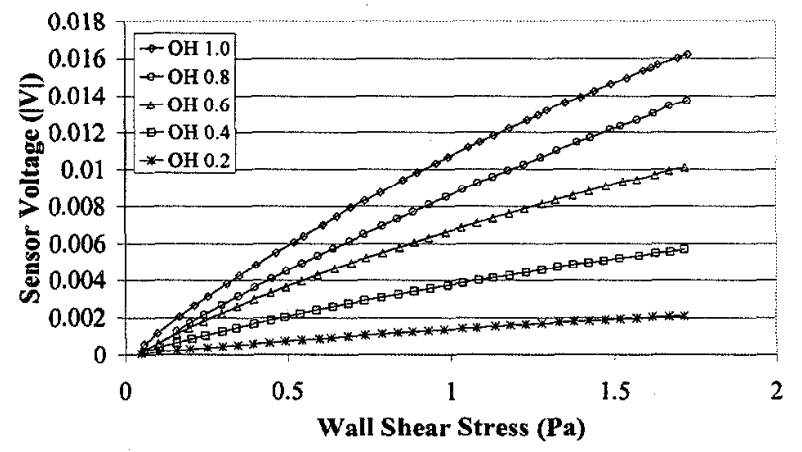

Figure 5. Change in sensor voltage as a function of wall shear stress at multiple thermal overheats for the sensor with vacuum cavity (4th order polynomial curve fits included).

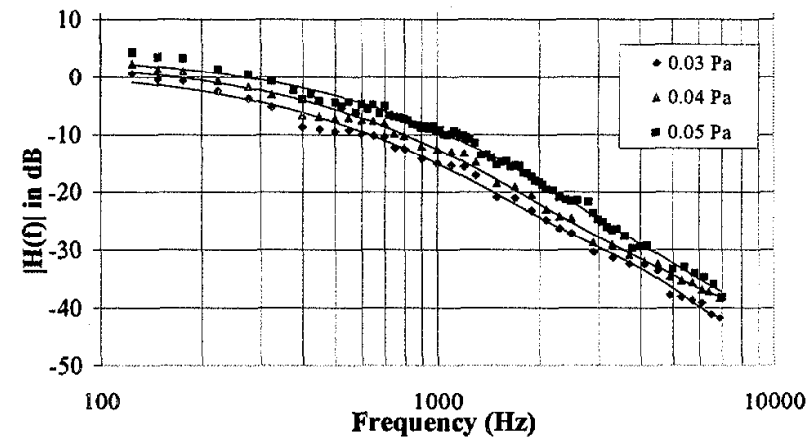

Figure 6. Frequency response function of the shear stress sensor at an overheat of 0.81 for multiple mean shear stress levels (4th order polynomial curve fits included). 


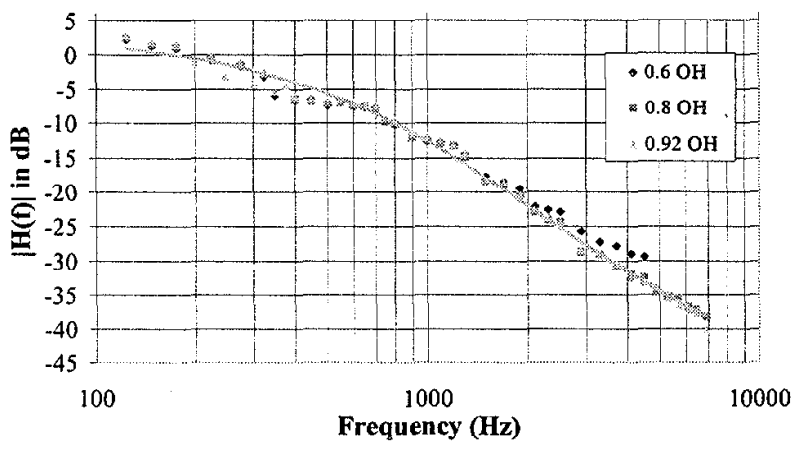

Figure 7. Frequency response function of the shear stress sensor at a mean shear stress of $0.04 \mathrm{~Pa}$ for multiple overheat ratios (4th order polynomial curve fits included).

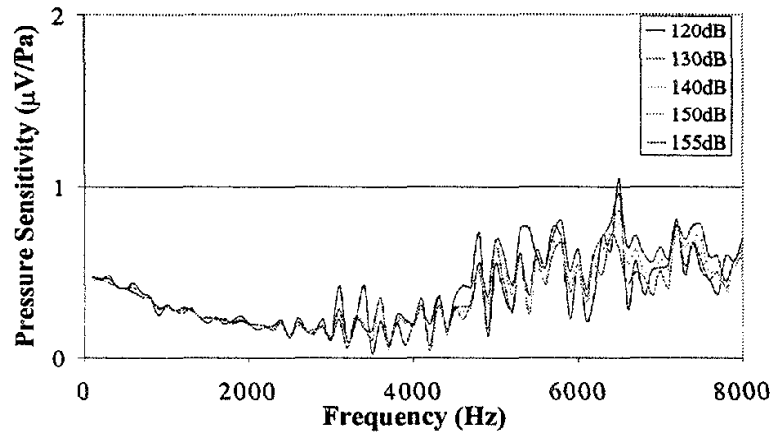

Figure 8. Dynamic pressure sensitivity spectrum of the shear stress sensor with cavity at multiple sound pressure levels ( $d B$ ref $20 \mu P a$ ).

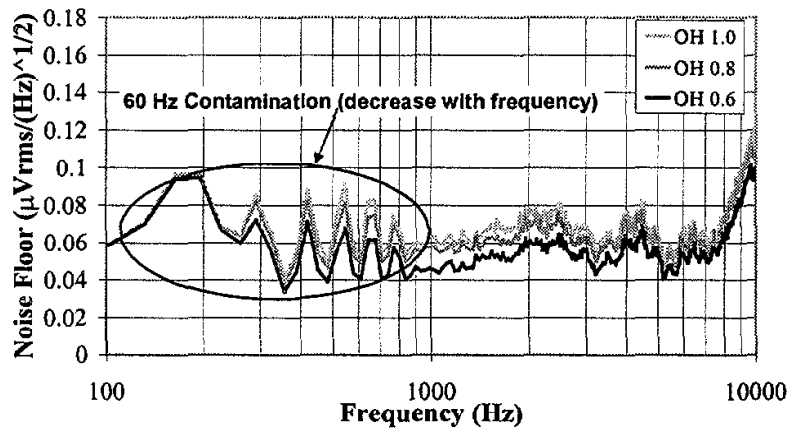

Figure 9. Measured noise spectrum of the shear stress sensor at zero mean shear stress and multiple overheats.

\section{CONCLUSIONS AND FUTURE WORK}

A thermal shear stress sensor for wind-tunnel measurements has been developed. The devices were fabricated using a novel wafer-bonding process that permits the fabrication of a sealed cavity thermal isolation structure. Thorough characterization of the sensor in a four-point probe configuration to exclude the effects of the external circuitry has been performed, including an in-situ dynamic calibration. Noise floor measurements in the operational range of the sensor have been obtained. The dynamic range of operation of the sensor has been experimentally verified $(9 \mu P a-1.7 P a)$ and the FRF obtained from $100 \mathrm{~Hz}$ to $8 \mathrm{kHz}$. The sensitivity to pressure and its effect on the dynamic response has been shown to be negligible. Future work will focus on characterizing the sensor in a constant temperature mode of operation and investigating the effects of sensor orientation in a flow field.

\section{ACKNOWLEDGEMENTS}

Support for this work was provided by the Air Force Office of Scientific Research (contract \# F4962-97-1-0507). The sensors were fabricated in the Microsystems Technology Laboratories (MTL) at the Massachusetts Institute of Technology with assistance from Mr. Joel Voldman.

\section{REFERENCES}

1. J. H. Haritonidis, "The Measurement of Wall Shear Stress," in Advances in Fluid Mechanics Measurements, SpringerVerlag, (1989), pp. 229-261.

2. A. Padmanabhan, et al., "Micromachined Sensors for Static and Dynamic Shear Stress Measurements in Aerodynamic Flows," Technical Digest, Transducers '97, Chicago, IL, (1997), 137-140.

3. C. Liu, et al., "A Micromachined Flow Shear-Stress Sensor Based on Thermal Transfer Principles," JMEMS, Vol. 8, No. 1, Mar. (1999), pp. 90-99.

4. E. Kalvesten, "Pressure and Wall Shear Stress Sensors for Turbulence Measurements," Ph.D. Dissertation, Royal Institute of Technology, Stockholm, Sweden, (1996).

5. A. Padmanabhan, et al., "Wafer-bonded floating-element shear stress microsensor with optical position sensing by photodiodes," JMEMS, Vol. 5. No. 4, Dec. (1996), pp. 307-315.

6. T. Pan, et al., "Microfabricated Shear Stress Sensors, Part 1: Design and Fabrication," AlAA Journal, Vol. 37, No. 1, Jan. (1999).

7. V. A. Sandborn, Resistance Temperature Transducers, Metrology Press, Fort Collins, Co., (1972).

8. B. J. Bellhouse, et al., "The Determination of Fluctuating Velocity in Air with Thin Film Gauges," J. Fluid Mech., Vol. 29, No.2, (1967), pp. 289-295.

9. S-L Jang, "A Model of $1 / \mathrm{f}$ Noise in Polysilicon Resistors," Solid-State Electronics, Vol. 33, (1990), pp. 1155-1162.

10. L. M. Fingerson, et al., "Thermal Anemometers," in Fluid Mechanics Measurements, R. J. Goldstein, Ed., Hemisphere, New York, (1983) pp. 99-154.

11. F. M. White, Viscous Fluid Flow, McGraw-Hill, NY, (1974), pp. 123-124.

12. B. J. Bellhouse, et al., "The Measurement of Fluctuating Skin Friction in Air with Heated Thin-Film Gauges," $J$. Fluid Mech, Vol. 32, part 4, (1968), pp. 675-680.

13. M. Sheplak, et al., " Dielectrically-Isolated, Single-Crystal Silicon, Piezoresistive Microphone," Technical Digest, Solid-State Sensor and Actuator Workshop, Hilton Head, SC, 23-26, June 1998.

14. M. A. Huff, et al., "Design of Sealed Cavity Microstructures Formed by Silicon Wafer Bonding," JMEMS, Vol. 2, No. 2, June (1993), pp. 74-81.

15. T. J. Hanratty, et al., "Measurement of Wall Shear Stress," in Fluid Mechanics Measurements, R. J. Goldstein, Ed., Hemisphere, New York, (1983) pp. 99-154.

16. M. Sheplak, et al., "Dynamic Calibration of a Shear Stress Sensor using Stokes Layer Excitation," AIAA Paper 980585, (1998).

17. V. Chandrasekaran, et al., "Dynamic Calibration Technique for Thermal Shear Stress Sensors with Variable Mean Duct Flows," AIAA Paper 2000-0508.

18. S. C. Ling, et al., "The Hot Film Anemometer: A New Device for Fluid Mechanics Research," J. Aero. Sci, 23, (1956), pp. 890-891. 